San Jose State University

SJSU ScholarWorks

Master's Theses

Master's Theses and Graduate Research

Fall 2020

\title{
A Meta-Analysis of fMRI Studies on Emotion Processing in Major Depressive Disorder
}

Madison Morocco

San Jose State University

Follow this and additional works at: https://scholarworks.sjsu.edu/etd_theses

\section{Recommended Citation}

Morocco, Madison, "A Meta-Analysis of fMRI Studies on Emotion Processing in Major Depressive Disorder" (2020). Master's Theses. 5156.

DOI: https://doi.org/10.31979/etd.jdd2-m4k3

https://scholarworks.sjsu.edu/etd_theses/5156

This Thesis is brought to you for free and open access by the Master's Theses and Graduate Research at SJSU ScholarWorks. It has been accepted for inclusion in Master's Theses by an authorized administrator of SJSU ScholarWorks. For more information, please contact scholarworks@sjsu.edu. 


\title{
A META-ANALYSIS OF FMRI STUDIES ON EMOTION PROCESSING IN MAJOR DEPRESSIVE DISORDER
}

\author{
A Thesis \\ Presented to \\ The Faculty of the Department of Psychology \\ San José State University \\ In Partial Fulfillment \\ of the Requirements for the Degree \\ Master of Arts
}

by

Madison Morocco

December 2020 
(C) 2020

Madison Morocco

ALL RIGHTS RESERVED 
The Designated Thesis Committee Approves the Thesis Titled

\title{
A META-ANALYSIS OF FMRI STUDIES ON EMOTION PROCESSING \\ IN MAJOR DEPRESSIVE DISORDER
}

\author{
by \\ Madison Morocco \\ APPROVED FOR THE DEPARTMENT OF PSYCHOLOGY \\ SAN JOSÉ STATE UNIVERSITY
}

December 2020

Dr. Valerie Carr

Dr. Matthew Capriotti

Dr. Erin Woodhead
Department of Psychology

Department of Psychology

Department of Psychology 


\title{
ABSTRACT \\ A META-ANALYSIS OF FMRI STUDIES ON EMOTION PROCESSING IN MAJOR DEPRESSIVE DISORDER
}

\author{
by Madison Morocco
}

The processing of an emotional stimulus involves a multi-step process that includes appraising and identifying a stimulus as well as producing an affective state in response. Many individuals with depressive disorders such as major depressive disorder (MDD) experience impairments related to emotion processing, likely caused by changes in the structure and function of brain regions important for emotion processing. However, the precise neural differences underlying emotion processing impairments in MDD remain unclear given conflicting findings in the neuroimaging literature. This lack of clarity has hindered the development of novel neurostimulation treatments for MDD, which require targeting of specific brain areas. In an effort to better identify specific areas of the brain to target for stimulation treatment, I conducted a meta-analysis of the functional magnetic resonance imaging (fMRI) literature to date regarding emotion processing in MDD. The meta-analysis of fMRI studies examined emotion processing in healthy controls and individuals with MDD using the tool GingerALE. Results showed clusters of hyperactivity in the amygdala and portions of the ventral basal ganglia, a finding that held true when limiting the analysis to only those studies that utilized negative stimuli. Additional exploratory results showed a cluster of hypoactivity in the right dorsolateral prefrontal cortex. These results help to shed light on neural changes underlying emotion processing in MDD, and may serve to inform future neurostimulation clinical trials. 


\section{ACKNOWLEDGMENTS}

I'd like to thank the many wonderful people who have encouraged and supported me throughout this program. I am extremely grateful for the extraordinary professors and advisors without whom this thesis would not have been possible. First, I'd like to thank Dr. Valerie Carr for her incredible guidance, support, and feedback. The amount of effort she puts toward her students is beyond any mentor I've had before. Thank you, Valerie, for caring so much and making your students such a priority. You're one of the best. Second, thank you to Dr. Matthew Capriotti and Dr. Erin Woodhead who were the first two professors I spoke with the summer before I began this program. I so appreciate the time and energy you both have given to supporting me in my journey from the very beginning. Your feedback and support have been invaluable.

Thank you to all three of you, Valerie, Matt, and Erin, for being wonderful examples of mentors and professors. There could not have been better people to work with in my time at San Jose State.

Thank you to my parents who encourage me to have high expectations for myself and who always help me achieve them. Your constant love and support means the world to me.

To my grandparents, I always want to make you proud. Thank you for your love, support, and for being such wonderful people to aspire to be. 


\section{TABLE OF CONTENTS}

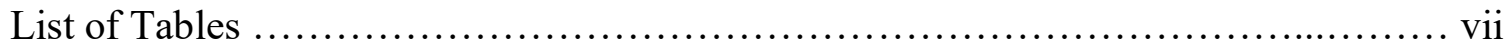

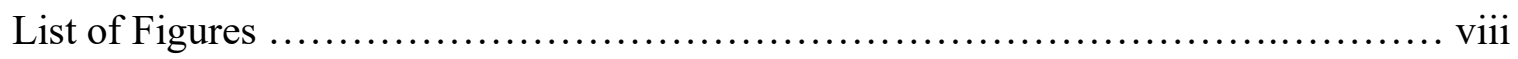

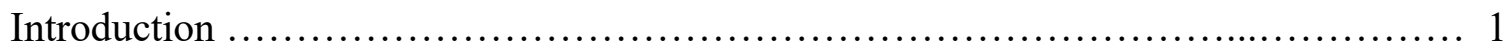

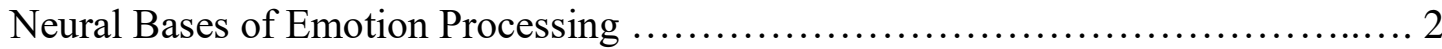

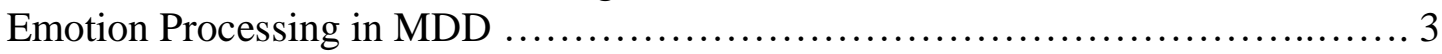

Aim and Hypothesis ............................................................ 7

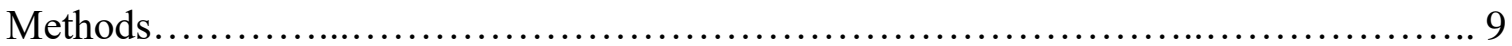

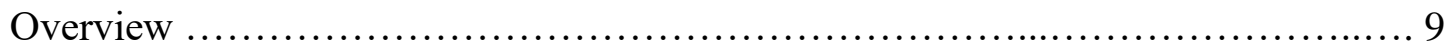

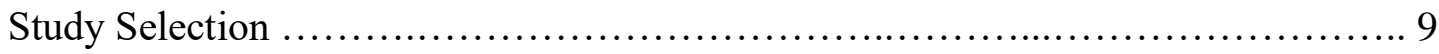

Meta-Analytics Procedure ............................................... 14

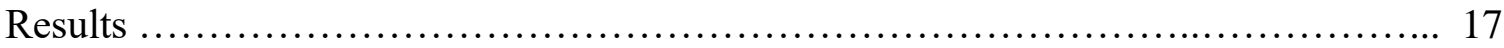

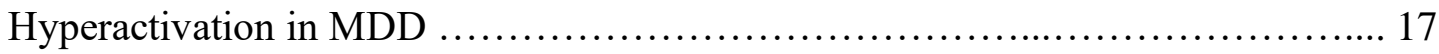

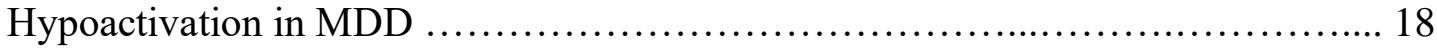

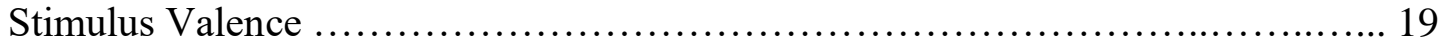

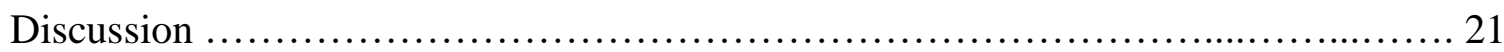

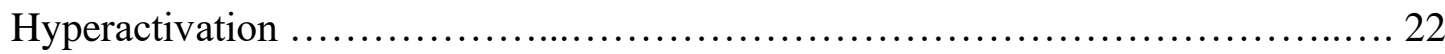

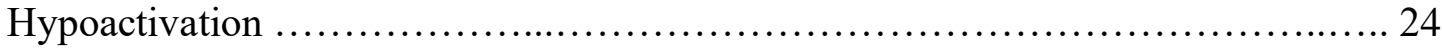

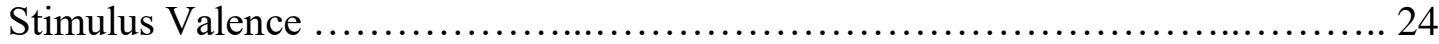

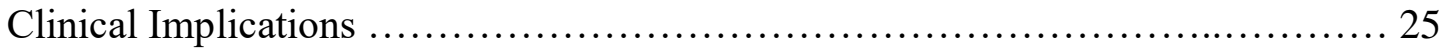

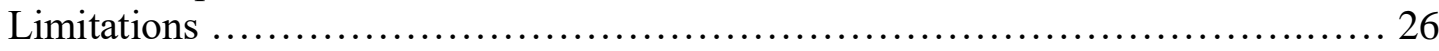

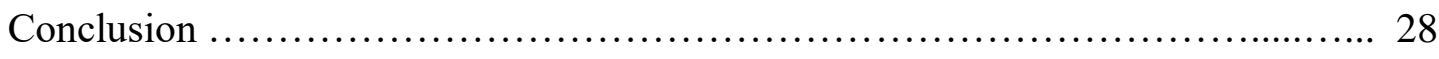

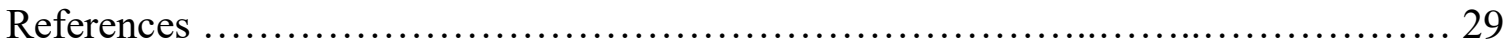




\section{LIST OF TABLES}

Table 1. Studies Included in Meta-Analysis ................................... 10 


\section{LIST OF FIGURES}

Figure 1. Cluster of activation for the stringent MDD $>\mathrm{HC}$ contrast $\ldots \ldots \ldots \ldots \ldots \ldots \ldots \ldots$

Figure 2. Clusters of activation for the exploratory MDD $>\mathrm{HC}$ contrast $\ldots \ldots \ldots \ldots \ldots . . .18$

Figure 3. Cluster of activation for the exploratory HC $>$ MDD contrast $\ldots \ldots \ldots \ldots \ldots \ldots$

Figure 4. Clusters of activation for the stringent Negative $>$ Neutral contrast ......... 20 


\section{Introduction}

Major depressive disorder (MDD) is a depressive disorder that affects approximately $20 \%$ of the U.S. population at some point in their lives, making it one of the most prevalent of all psychiatric disorders (Joormann \& Quinn, 2014). MDD is characterized by several symptoms that can greatly affect an individual's quality of life (Wang et al., 2019). Symptoms of MDD include low mood, fatigue, loss of interest in activities, and poor emotion processing (Park, et al., 2019; Wang et al., 2019). Indeed, MDD has a negative impact on quality of life, such that MDD is a leading global cause of disability (Murray et al., 2015).

Although numerous studies have demonstrated alterations in emotion processing in MDD, the neural mechanisms underlying these alterations remain unclear, making the development of novel treatments challenging (Stuhrmann, Suslow, \& Dannlowski, 2011). There are several types of efficacious treatments for MDD, including specific psychotherapies and pharmacotherapy (Wang et al., 2019), but 50\% of individuals with MDD do not respond to these treatments (Akechi et al., 2020), and therefore experience a continued negative impact on quality of life (Wang et al., 2019).

For individuals with treatment resistant depression (TRD), newer types of treatment are being evaluated in clinical trials, including forms of neural stimulation such as deep brain stimulation (DBS) and transcranial magnetic stimulation (TMS), as well as intranasal ketamine (Pradhan, Parikh, Makani, \& Sahoo, 2015). Whereas ketamine has systemic effects, DBS and TMS require targeted stimulation of specific brain areas. Preliminary findings from neurostimulation trials have revealed mixed findings in terms 
of the effectiveness of DBS and TMS (Filkowski \& Sheth, 2019; Wang, 2019), such that more research is needed to identify optimal areas to target for treatment. A recent review by Filkowski and Sheth (2019) suggests that gaps in the literature have contributed to the field's inability to identify specific areas of the brain to target for stimulation treatment in TRD. In an effort to address this issue, I performed a meta-analysis of functional MRI (fMRI) studies comparing brain activity during emotion processing in individuals with MDD and healthy controls to discern valuable information regarding areas of dysfunction in MDD, and thus potential areas for treatment.

\section{Neural Bases of Emotion Processing}

Emotional responses involve a complicated, multi-dimensional process that changes over time with respect to latency, rise time, magnitude, and duration (Gross, 2002). We may feel negative emotions after hearing a hurtful comment, positive emotions after witnessing an awe-inspiring moment, or neutral emotions after relaxing. Regardless of the kind of emotion, these coordinated behavioral, experiential, and physiological response tendencies influence how we respond to our perceived challenges and opportunities (Gross, 2002). With respect to the neuropsychological basis of such responses, Phillips, Drevets, Rauch, and Lane (2003) propose that the following processes occur after exposure to an emotional stimulus: (1) appraisal and identification of the emotional significance of the stimulus, (2) production of a specific affective state in response to the stimulus, and (3) regulation of the affective state and emotional behavior. For the purposes of this meta-analysis, I focused on neural processes supporting the first two stages, henceforth referred to as emotion processing. 
Previous neuroimaging research has linked emotion processing to several areas of the prefrontal cortex and limbic system. Specifically, these areas include the medial prefrontal cortex, dorsolateral prefrontal cortex, ventrolateral prefrontal cortex, anterior cingulate cortex, orbitofrontal cortex, and the amygdala (Ahmed, Bittencourt-Hewitt, \& Sebastian, 2015; Yoo, Gujar, Hu, Jolesz, \& Walker, 2007). Each of these regions plays a role in emotion processing, whether it be expressing emotions (medial prefrontal cortex and anterior cingulate cortex) or the emotionality of reward-based processing (e.g., gambling; orbitofrontal cortex) (Ahmed et al., 2015; Barbas, 2007; Blakemore, 2008; Davidson, Putnam, \& Larson, 2000; Etkin, Egner, \& Kalisch, 2011; Gasquoine, 2013). Additionally, the amygdala has been linked to emotion regulation, emotion response, emotional memory, and emotional learning in healthy individuals (LeDoux, 2000). Given the significance of the prefrontal cortex and limbic system in emotion processing, and given that impaired emotion processing is a common symptom of MDD, many researchers have examined whether and how activity in these areas differs in individuals with MDD relative to healthy controls.

\section{Emotion Processing in MDD}

Impaired emotion processing in MDD can manifest in a variety of ways. For example, individuals with MDD tend to rate negative stimuli as more negative than healthy individuals (Fonseka, Jaworska, Courtright, MacMaster, \& MacQueen, 2016), and they have increased accessibility of negative memories and decreased accessibility of positive memories (Joormann \& Quinn, 2014). Further, multiple studies show that individuals with MDD are more likely to remember negative words and negative 
autobiographical memories than positive words and positive autobiographical memories (Joormann \& Quinn, 2014; Matt, Vázquez, \& Campbell, 1992). These cognitive biases suggest that individuals with MDD may show abnormal patterns of activity in the abovementioned regions supporting emotion processing (Gotlib \& Joormann, 2010; Kaiser et al., 2015).

fMRI studies of emotion processing in people with MDD typically involve examining brain activity in response to emotional vs. neutral stimuli (Canli et al., 2004; Epstein et al., 2006; Lang, Bradley, \& Cuthbert, 1997; Sacchet et al., 2017). For example, participants would view emotional images (e.g., angry or crying people, spiders, or a cemetery) as well as neutral images (e.g., a wall or geometric shapes), and neural responses to each image type would be compared in individuals with MDD as well as healthy controls. Studies using this approach have reported dysfunctional activity in MDD in both the amygdala and prefrontal cortex in individuals. Studies using negative images from the International Affective Picture System (IAPS;Lang, Bradley, \& Cuthbert, 1997) have reported greater activity (i.e., hyperactivity) in MDD relative to controls in several regions of the frontal lobe (supplemental motor area, bilateral prefrontal cortex, dorsolateral prefrontal cortex, right lateral orbitofrontal cortex, right medial orbitofrontal cortex) as well as the dorsal anterior cingulate cortex, right middle temporal gyrus, amygdala, insula, and thalamus (Davis et al., 2019; Johnstone et al., 2007; Rosenblau et al. 2012; Tozzi et al., 2017).

Another common task, called the Facial Emotion Perception Test (FEPT), features images of people making emotional or neutral facial expressions in which participants are 
asked to categorize each face by emotion (anger, happiness, sadness, fear, or neutral) using a 5-button response box (Jenkins et al., 2017). Brain activity in response to each image type is then compared in individuals with MDD and controls. An fMRI study using the FEPT demonstrated hyperactivity in the amygdala, insula, and subgenual anterior cingulate cortex in MDD relative to controls when viewing emotional faces (Jenkins et al., 2017), findings that are consistent with some, but not all, of the abovementioned results.

Another commonly used task involves presenting participants with sets of emotional and neutral words or word pairs (Canli et al., 2004; Epstein et al., 2006; Sacchet et al., 2017). Results have demonstrated hyperactivity in the right middle frontal gyrus, amygdala, hippocampus, and inferior parietal lobule in MDD relative to controls when processing negative emotional stimuli (Canli et al., 2004; Sacchet et al., 2017). However, individuals with MDD demonstrated less activity (i.e., hypoactivity) than controls when processing positive emotional stimuli in the bilateral ventral striatal, dorsomedial frontal region, superior temporal gyrus, cerebellum, and dorsolateral prefrontal cortex (Canli et al., 2004; Epstein et al., 2006). The dorsolateral prefrontal cortex, in particular, is a disputed region as it has been shown to be both hyper- and hypoactive in individuals with MDD compared to HC in different studies (Groenewold, Opmeer, de Jonge, Aleman, \& Costafreda, 2013; Rosenblau et al. 2012).

Several reviews of this literature have been published (e.g., Dichter, Gibbs, \& Smoski, \& 2015; Jaworska, Yang, Knott, MacQueen, \& 2015; Rive et al., 2013; Stuhrmann et al., 2011; Young et al., 2018), as well as one prior meta-analysis of emotion 
processing in MDD (Groenewold et al., 2013). Groenewold et al. (2013) found that participants with MDD showed dysfunction in the amygdala, striatum, parahippocampal cortex, cerebellum, fusiform gyrus, and anterior cingulate cortex during emotion processing tasks. Interestingly, the direction of the dysfunction (i.e., hyper- or hypoactivation in MDD relative to controls) depended on the valence of the stimuli. For example, viewing negatively-valenced stimuli resulted in hyperactivation of these regions in MDD, while viewing positively-valenced stimuli resulted in hypoactivation of the same regions. Critically, in the time since this meta-analysis was published, multiple relevant fMRI studies have been reported, creating an opportunity to repeat these analyses with newer data and a larger sample size.

Taken together, studies of emotion processing in MDD have revealed areas of both increased and decreased neural activity relative to controls. Across several studies, the amygdala, insula, frontal cortex, and cingulate cortex areas have been shown to be hyperactive in MDD relative to controls. Conversely, a small number of studies have indicated that the superior temporal gyrus, cerebellum, bilateral ventral striatal, and dorsomedial frontal areas are hypoactive in MDD relative to controls. It also bears noting that several other areas (e.g., supplemental motor area, right middle temporal gyrus, thalamus, right inferior frontal sulcus) have been reported to show dysfunction in MDD, but such findings come from single studies and have not been replicated.

Given the lack of consistency in the literature to date, greater clarification regarding areas of dysfunction during emotion processing in MDD is needed to better inform clinical trials of DBS and TMS, potentially improving treatment efficacy and thereby the 
patients' quality of life (Kisely, Li, Warren, \& Siskind, 2018; Liu, Sheng, Li, \& Zhang, 2017). To address this issue, I conducted a meta-analysis of functional neuroimaging studies of emotion processing in MDD to synthesize the current literature and determine whether one or more regions are consistently shown to be hyper- and/or hypoactive in this population.

\section{Aim and Hypothesis}

Since Groenewold et al.'s meta-analysis was published in 2013, more than 20 emotion processing studies have been published that have the potential to better inform current understanding of brain regions exhibiting dysfunction in MDD during emotion processing. As such, I conducted an fMRI meta-analysis of emotion processing in MDD inclusive of fMRI studies published through 2020. Studies pertaining to emotion regulation were excluded given that the tasks used are quite different. Such studies typically use stimuli similar to those in emotion processing studies, but participants are asked to actively regulate their emotions in response to these the stimuli instead of passively view them. As more fMRI studies of emotion regulation in MDD are published in the future, researchers can conduct meta-analyses of this literature.

A meta-analysis not only synthesizes vast amounts of research, but critically, it also allows for statistical analysis of prior findings and provides quantitative results, unlike a qualitative review (Gravetter, Wallnau, Forzano, \& Witnauer, 2020). In the current study, I aimed to answer the following questions: (1) in fMRI studies of emotion processing in MDD, which regions consistently demonstrate impairments in emotion processing?, (2) for each of these areas, do individuals with MDD demonstrate greater or lesser activity 
than controls when processing emotional stimuli, (3) are these effects related to stimulus valence?

Based on a qualitative review of the fMRI literature and the meta-analytic findings of Groenewold et al. (2013), I hypothesized that prefrontal cortex regions, the anterior cingulate cortex, and the amygdala would be consistently more active in patients relative to controls when performing an emotion processing task. Further, I hypothesized that these findings would hold true when including only those studies that focused on negative stimuli, specifically. Lastly, I hypothesized that the dorsomedial frontal regions, the dorsolateral prefrontal cortex, and bilateral ventral striatal would be consistently less active in patients relative to controls when performing an emotion processing task. 


\section{Methods}

\section{Overview}

I conducted a meta-analysis of the fMRI literature to date regarding emotion processing in MDD using GingerALE (http://www.brainmap.org; Laird, Lancaster, Fox, \& Uecker, 2003), a software program for manually performing fMRI meta-analyses.

\section{Study Selection}

To select studies to be included, I conducted a literature review using the databases PsycINFO and PubMed and the search terms "Major Depressi(*) AND emotion processing AND fMRI". Relevant papers were included that met the following criteria:

(1) studies that compare individuals with a current MDD diagnosis (no comorbid disorders) to individuals in a healthy control group, (2) studies that use an emotion processing task that involves both emotional and neutral stimuli, and (3) studies that use participants that are young to middle-aged adults $(18-60)$. The latter was chosen to avoid confounds associated with structural and functional differences in older adults. My literature review indicated that 30 studies met these criteria, published between 2004 and 2019. Table 1 includes a list of these studies along with the number of participants in each group, specific task and stimuli used, conditions compared for statistical purposes, and reference space used. 


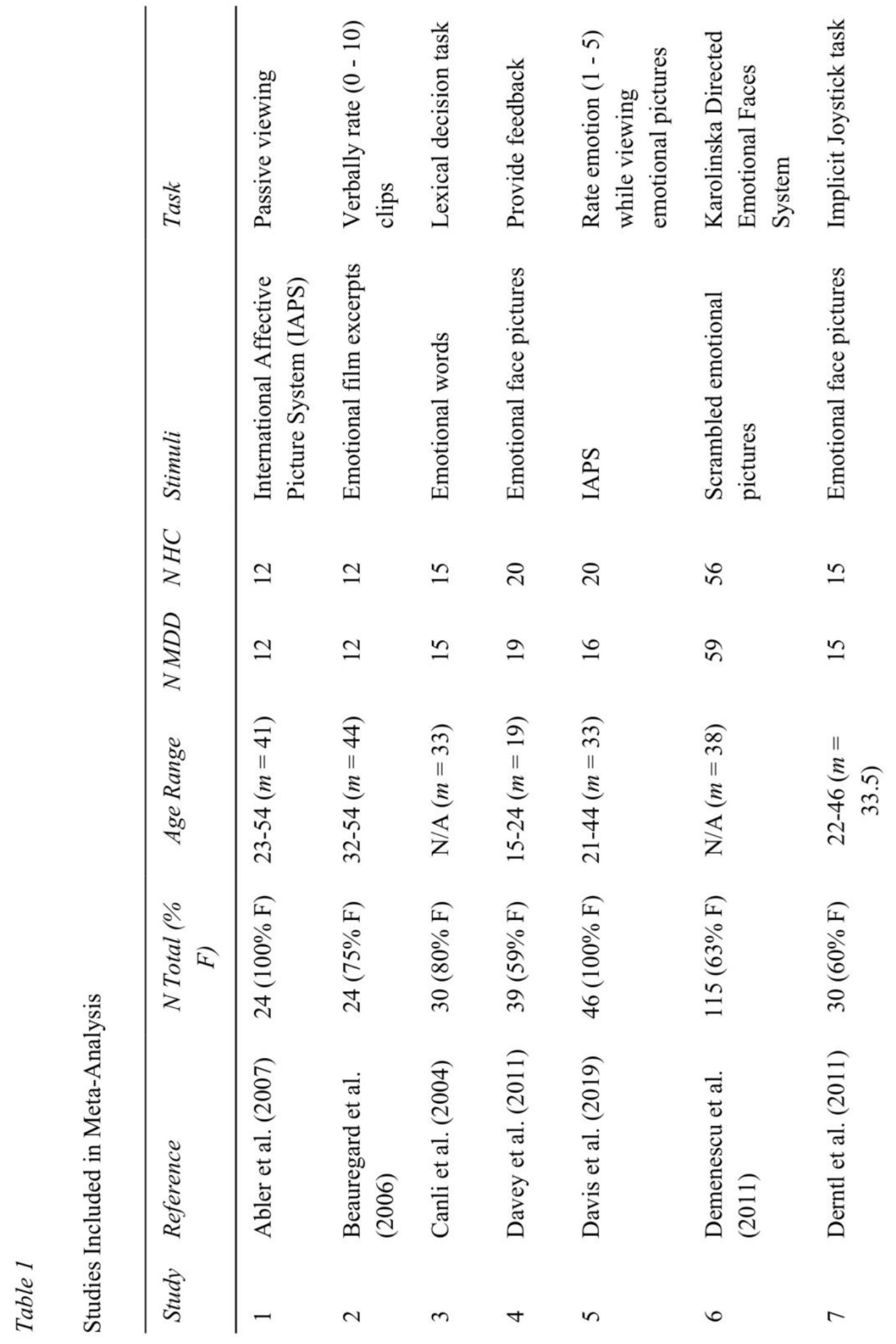




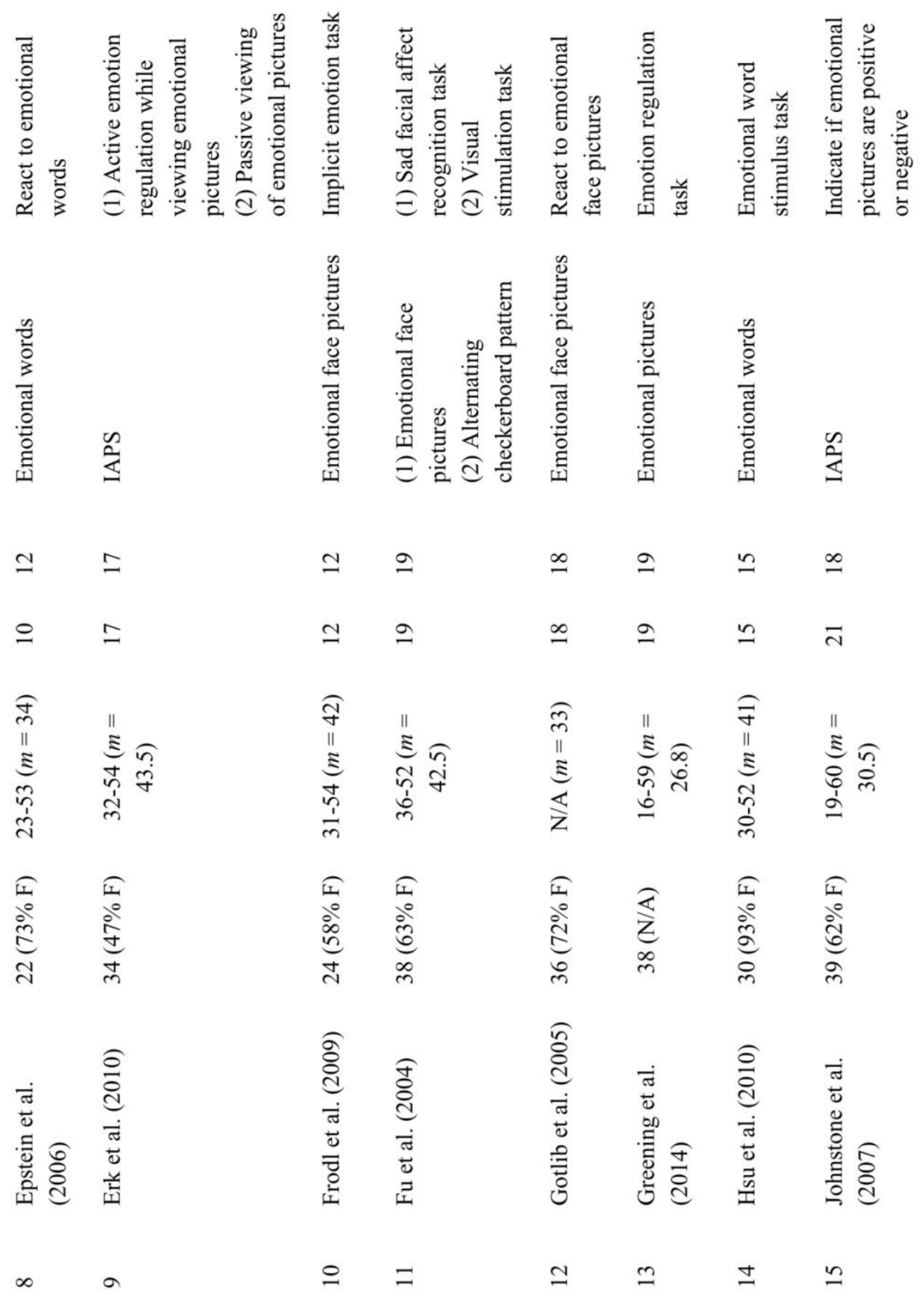



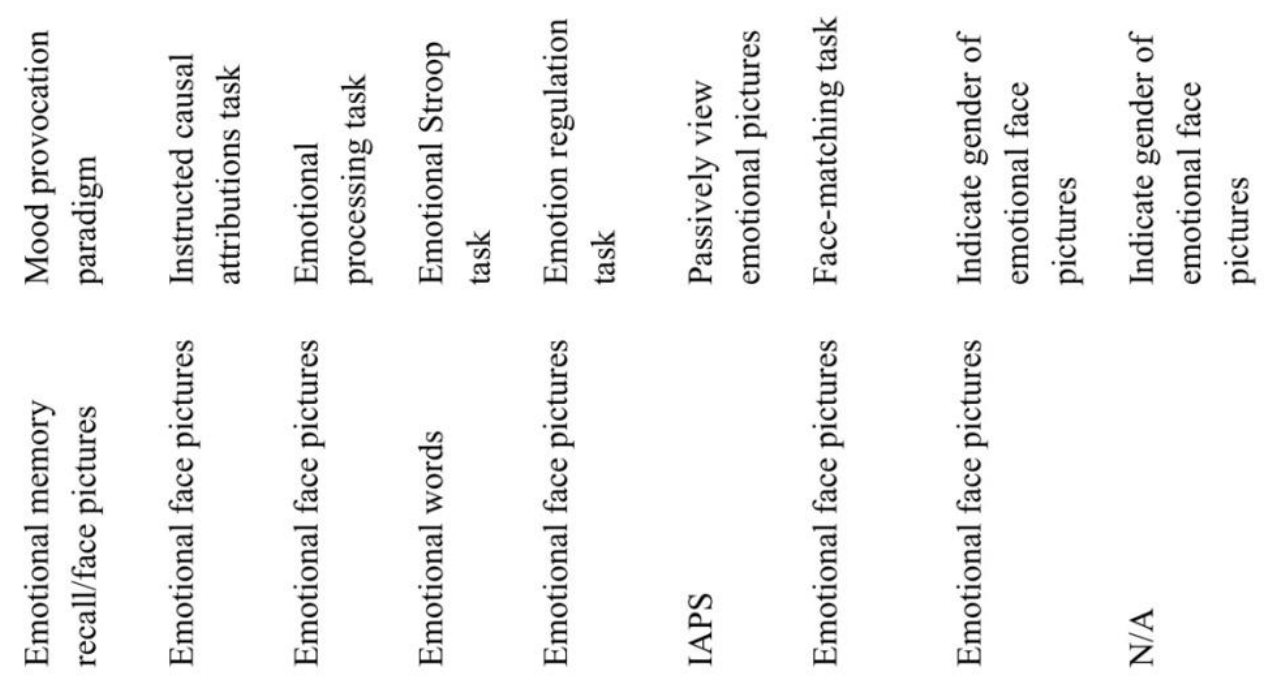

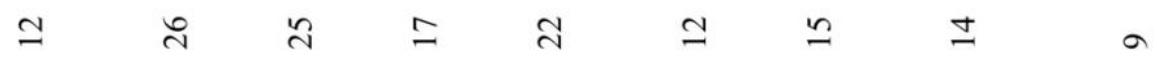

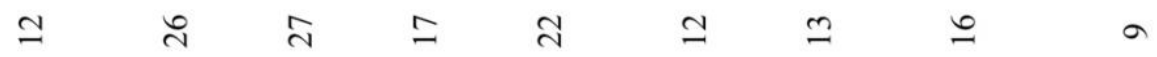

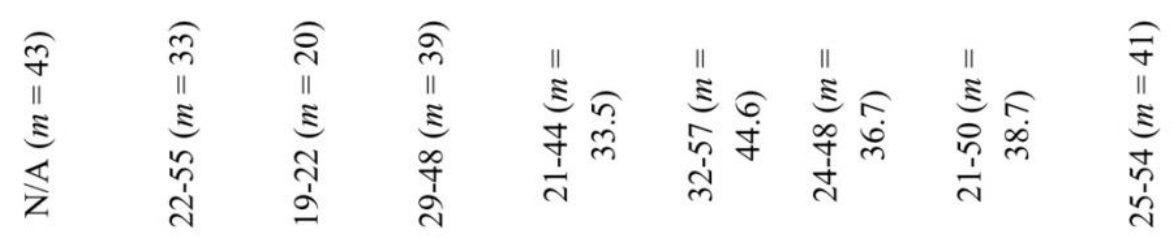

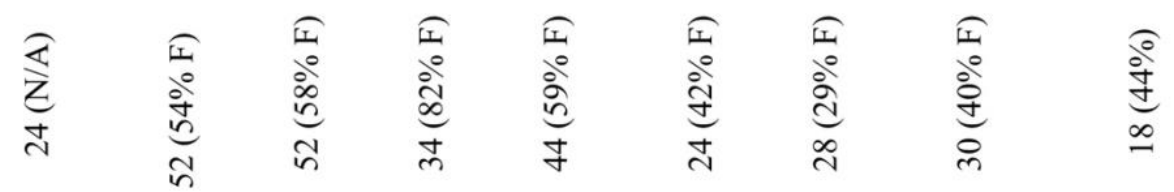

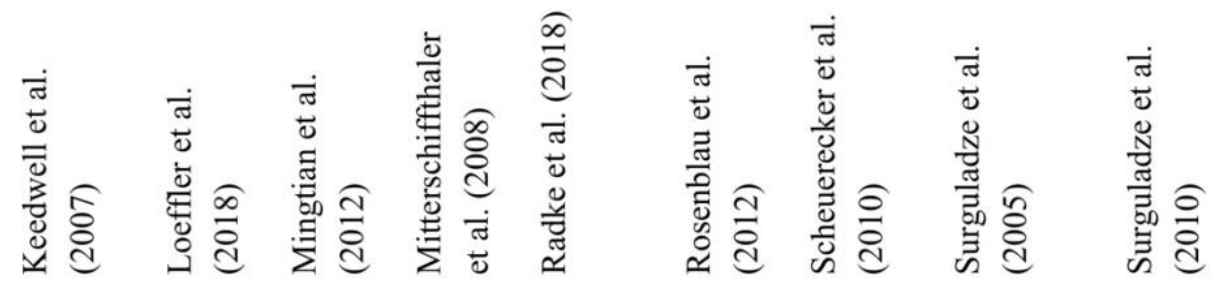

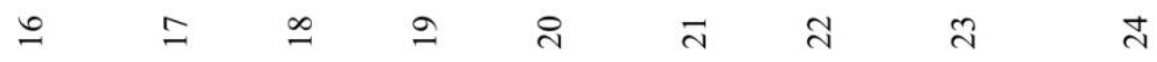




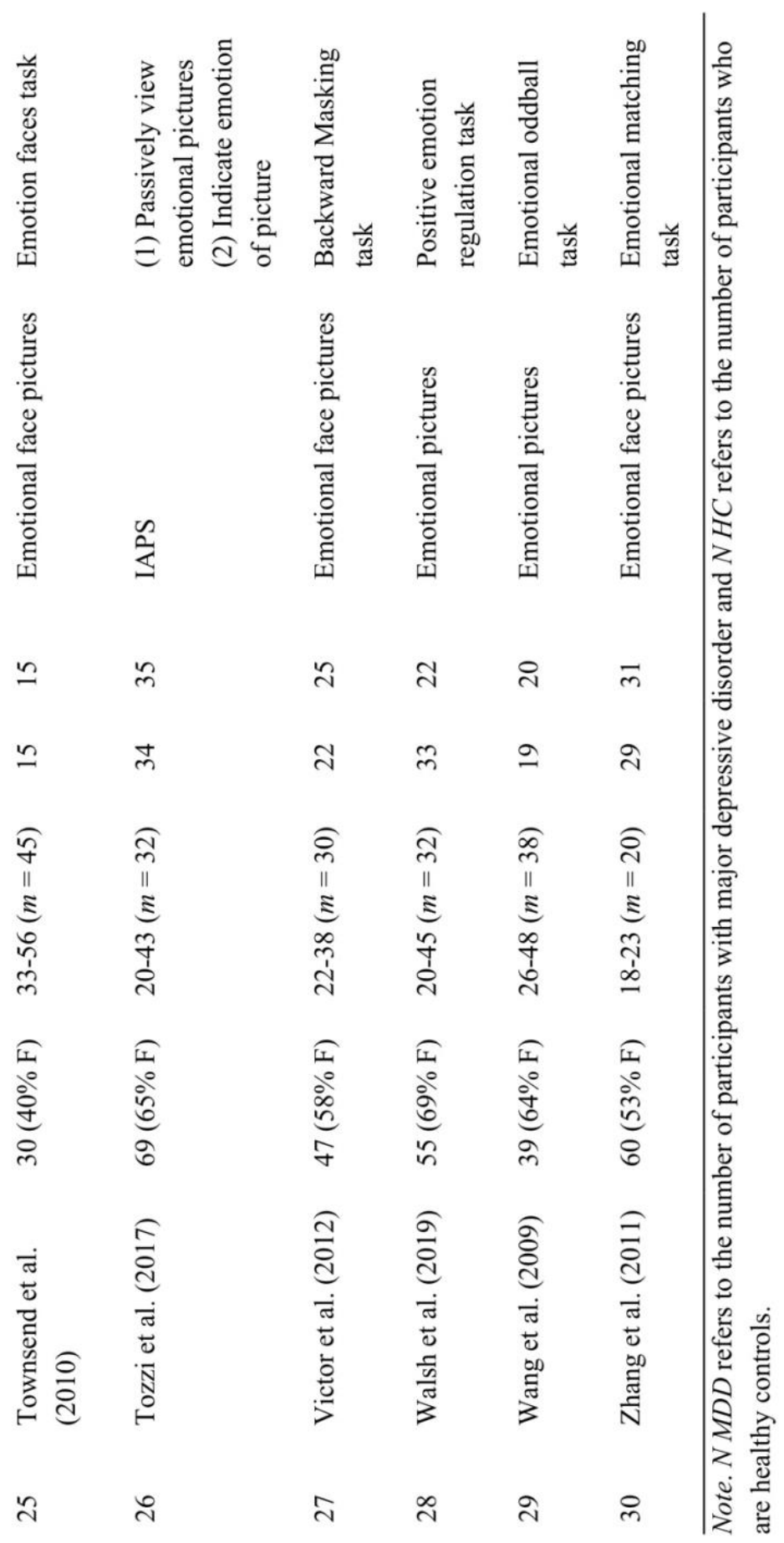




\section{Meta-Analytic Procedure}

GingerALE is a free software tool for manually conducting fMRI meta-analyses. This rigorous method requires the user to (1) search for papers that meet strict, user-defined criteria, (2) extract activity peaks from desired analyses in each paper, (3) convert activity peak coordinates to a common reference space, and (4) choose statistical settings for running the analysis (Laird et al., 2005). With respect to step (3), each person's brain is a slightly different shape and size, to which end fMRI studies "normalize" participant brains to one of two common reference spaces: Talairach or MNI (Montreal Neurological Institute) space. MNI is a newer reference space that was created using a combination of hundreds of brains, making it more representative of the general public than Talairach space, which is based on a single individual's brain. For the current study, all activity peaks were converted to MNI space.

GingerALE uses an activation likelihood estimate (ALE) for conducting the metaanalysis, which involves computing ALE values for each voxel (i.e., volumetric pixel) in the brain to determine whether activity in that voxel was consistently reported across studies (Laird et al., 2003). Significant clusters of activity are then displayed in an ALE map.

More specifically, GingerALE analyzes the spatial convergence of the peaks of activation for the studies as a set. First, GingerALE smooths the imperfect foci using a three-dimensional Gaussian function to get more accurate and useful estimates of the active regions. Next, these smoothed foci are put together into an ALE map which shows the pattern of activation across the set of studies selected for the meta-analysis. This map 
reflects the likelihood that each voxel in the brain truly represents activation. Lastly, GingerALE uses a statistical threshold, set by the user, to calculate the common patterns of activation across the set of studies. This ALE map is compared to another ALE map in which an equivalent number of peaks of activation are randomly generated. GingerALE compares the true pattern of activation to this random distribution to determine whether the pattern is more likely to be found than a chance distribution (Levy \& Wagner, 2011). I conducted two analyses on each set of data; the first a stringent analysis that corrects for multiple comparisons, and the second more exploratory. For the stringent analysis, I used a cluster-level family-wise error of $p<0.05$ to correct for multiple comparisons, following an initial cluster forming threshold of uncorrected $p<0.001$. For the exploratory analysis, I used a cluster forming threshold of uncorrected $p<0.001$ and a minimum cluster volume of $300 \mathrm{~mm}^{3}$. GingerALE analyses were performed using GingerALE version 2.3.6), using the standard default parameters (http://brainmap.org/ale/).

Using the above approach, I conducted three separate meta-analyses. The first examined areas of the brain showing hyperactivity in MDD relative to controls (MDD > HC) during emotion processing tasks, and the second examined areas of the brain showing hypoactivity in MDD relative to controls during such tasks (HC > MDD). I then repeated the first analysis (MDD > HC) to specifically focus on studies that evaluated responses to negative vs. neutral stimuli, i.e., I removed studies that utilized positive stimuli or a mix of positive and negative stimuli. Unfortunately, there were insufficient 
data to conduct a similar analysis focusing only on those studies utilizing positive vs. neutral stimuli (11 foci across 4 studies). 


\section{Results}

\section{Hyperactivation in MDD}

The first analysis used the contrast MDD > HC to examine areas of hyperactivation in MDD relative to controls when performing an emotion processing task. The analysis was based on 187 foci across 25 studies. Using a stringent threshold, this analysis revealed one significant cluster that covered the right amygdala and ventral portions of the putamen and globus pallidus (Figure 1). The exploratory analysis using a more lenient threshold revealed the same right amygdala cluster and additional clusters in the left amygdala and hippocampus, left nucleus accumbens, and right Brodmann areas 46 and 47 within the inferior frontal gyrus (Figure 2).

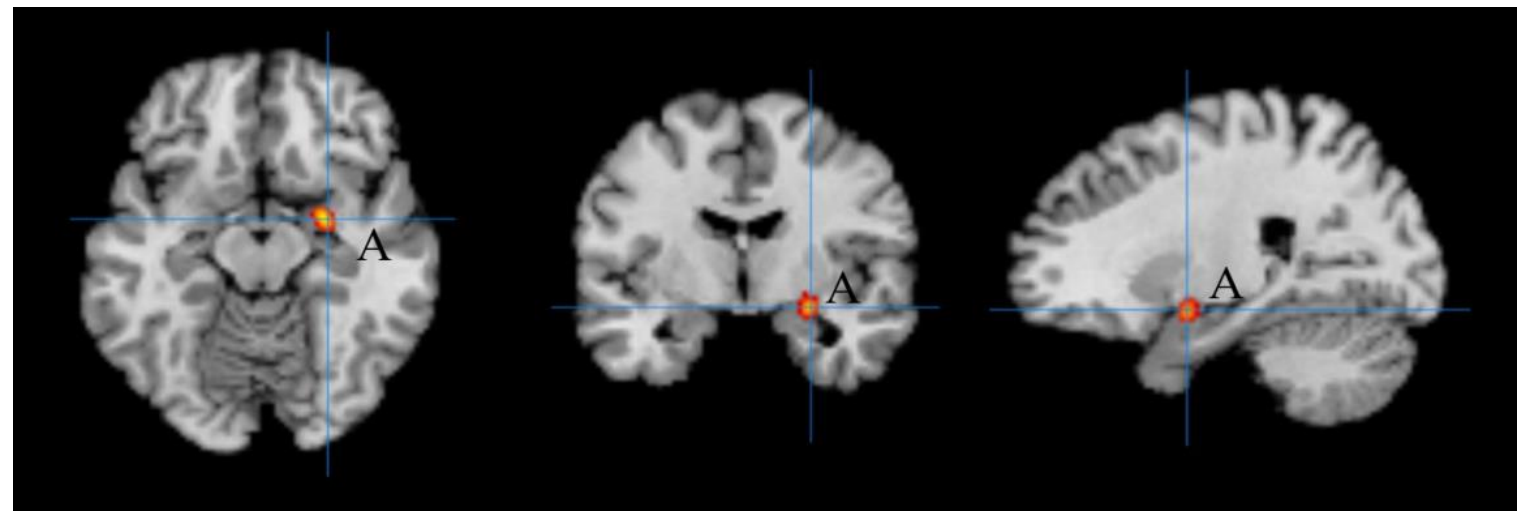

Figure 1. Cluster of activation for the stringent MDD > HC contrast. (A) denotes a significant cluster that covers the right amygdala and ventral portions of the putamen and globus pallidus. 


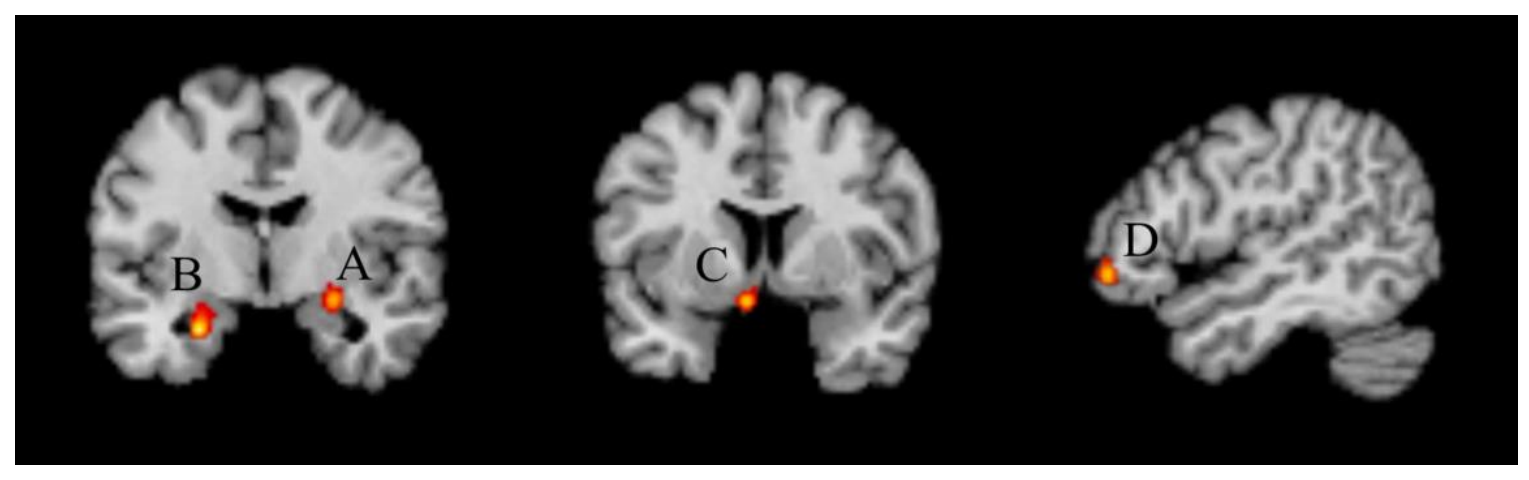

Figure 2. Clusters of activation for the exploratory MDD > HC contrast. (A) denotes the right amygdala and ventral portions of the putamen and globus pallidus, (B) denotes the left amygdala and hippocampus, (C) denotes the left nucleus accumbens, and (D) denotes Brodmann areas 46 and 47 in the right inferior frontal gyrus.

\section{Hypoactivation in MDD}

Next, I conducted a meta-analysis using the reverse contrast of $\mathrm{HC}>\mathrm{MDD}$ to examine areas that were hypoactive in individuals with MDD relative to controls when performing an emotion processing task. The analysis was based on 117 foci across 15 studies. Using a stringent threshold, this analysis did not reveal any significant clusters. However, an exploratory analysis using a more lenient threshold returned one significant cluster in Brodmann area 9 in the right dorsolateral prefrontal cortex (Figure 3).

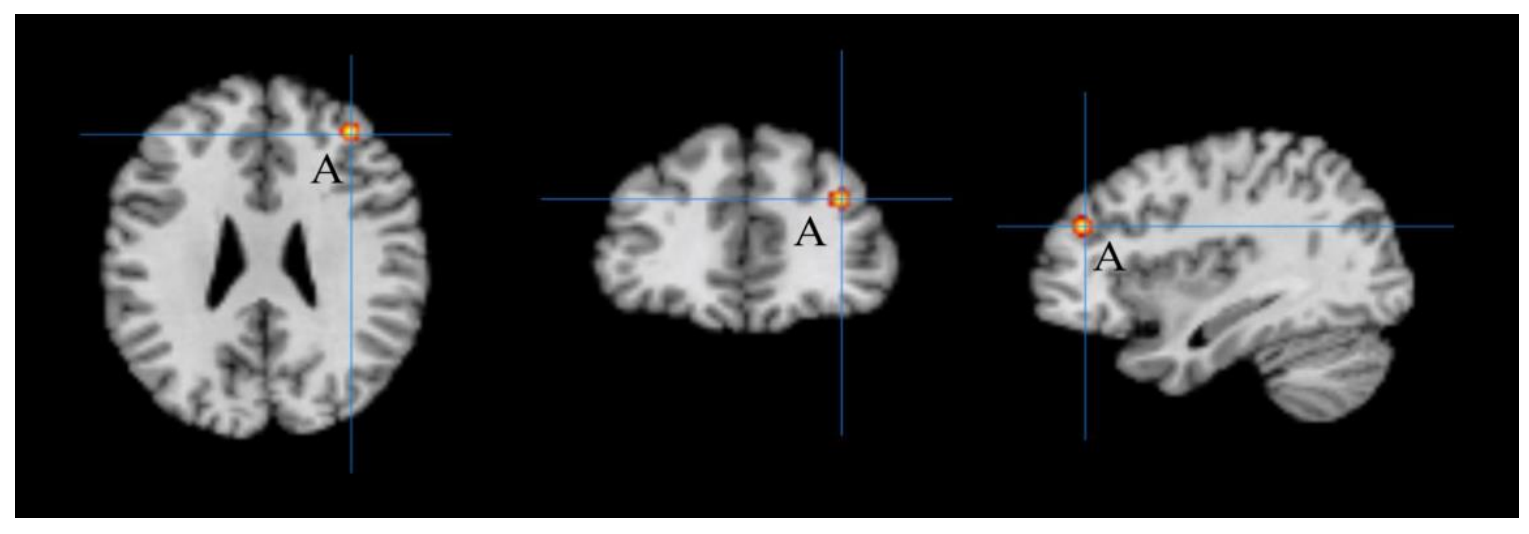

Figure 3. Cluster of activation for the exploratory HC > MDD contrast. (A) denotes one significant cluster in Brodmann area 9 in the right dorsolateral prefrontal cortex. 


\section{Stimulus Valence}

The remaining meta-analysis was designed to examine whether the observed hyperactivity in MDD relative to controls held when focusing specifically on those studies that evaluated responses to negative vs. neutral stimuli. This analysis included 37 foci across 7 studies. Using a stringent threshold, the analysis revealed two significant clusters: one in the left amygdala and parahippocampal gyrus, and the other in the right amygdala and ventral putamen (Figure 4). Using a more lenient threshold did not reveal any additional clusters. 


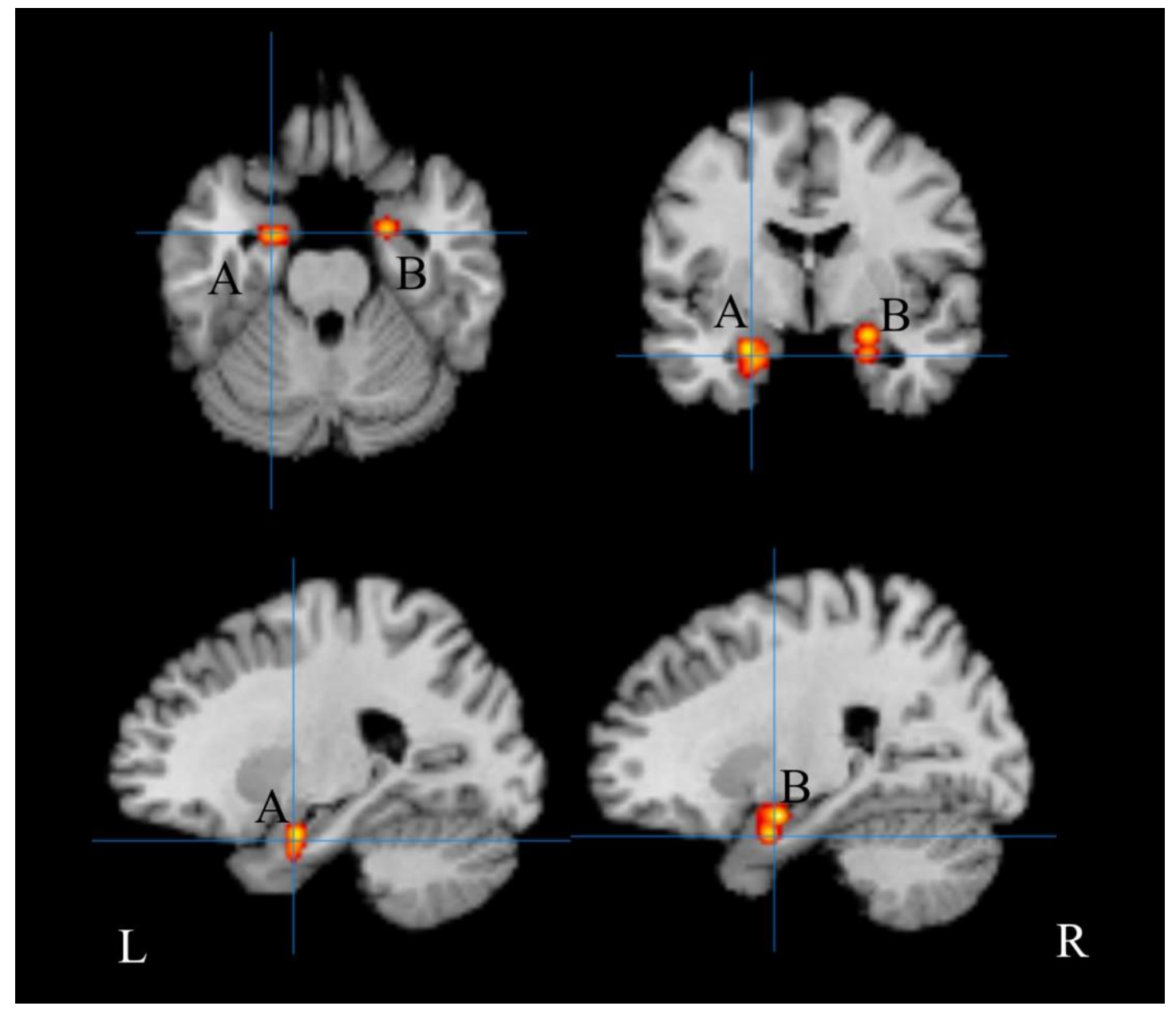

Figure 4. Clusters of activation for the stringent Negative $>$ Neutral contrast. (A) denotes the left amygdala and parahippocampal gyrus, and (B) denotes the right amygdala and ventral putamen. 


\section{Discussion}

This study involved meta-analysis of fMRI data comparing brain activity in individuals with MDD and healthy controls during emotion processing tasks. The overarching aim was to identify areas of dysfunction consistently reported across studies, and thus potential areas for treatment. A series of analyses revealed areas of both hyperactivation and hypoactivation in individuals with MDD relative to controls. The right amygdala and ventral portions of the putamen and globus pallidus were found to be consistently hyperactive across studies when using a stringent statistical threshold. A more lenient exploratory analysis revealed additional hyperactivity in the MDD group in the left amygdala and hippocampus, left nucleus accumbens, and right Brodmann areas 46 and 47 within the inferior frontal gyrus. These findings are consistent with my hypothesis that prefrontal cortex regions and the amygdala would be consistently more active in patients relative to controls when performing an emotion processing task. However, in contrast to my predictions, anterior cingulate cortex was not shown to be consistently hyperactive across studies. When repeating the hyperactivity meta-analysis to include only those studies utilizing negative vs. neutral stimuli, results revealed bilateral amygdala hyperactivity in MDD relative to controls. Finally, with respect to hypoactivity, no regions were found to be consistently less active in MDD than controls using a stringent threshold. However, an exploratory analysis revealed hypoactivity in the MDD group in Brodmann area 9 in the right dorsolateral prefrontal cortex. This finding somewhat supports my hypothesis; however, I did not find hypoactivity in the dorsomedial frontal regions nor bilateral ventral striatal, as hypothesized. 


\section{Hyperactivation}

The hyperactivity findings in the current study largely support those of a previous meta-analysis conducted by Groenewold et al. (2013), who reported peaks of hyperactivation in the amygdala, striatum, parahippocampus, cerebellum, fusiform, and anterior cingulate cortex in individuals with MDD compared to healthy controls. Using a stringent statistical approach, I report hyperactivity in individuals with MDD in the amygdala and ventral portions of putamen and globus pallidus, regions that are linked to emotion regulation, emotion response, emotional memory, and emotional learning (LeDoux, 2000). In healthy individuals, previous studies have identified activation of the amygdala, insula, and anterior temporal lobe during emotion processing tasks (Beauregard, Paquette, \& Le, 2006). However, as demonstrated by the current study, this amygdala activation is more extreme in individuals with MDD. It has been suggested that this hyperactivation is a product of reduced connectivity between the amygdala and a variety of brain regions involved in emotion processing and regulation, including the ventrolateral prefrontal cortex, insula, caudate, middle and superior temporal regions, occipital cortex, and cerebellum (Ramasubbu et al., 2014). Such reductions in connectivity may lead to dysfunction in bottom-up signaling, leading to altered emotional regulation.

The hyperactivity cluster centered in the right amygdala also included ventral portions of the basal ganglia, specifically the putamen and globus pallidus. Normal basal ganglia function is associated with emotion processing, among other functions like motor control (Paradiso, Ostedgaard, Vaidya, Ponto, \& Robinson, 2013). In healthy individuals, 
previous fMRI studies have demonstrated increased reactivity to emotional stimuli (Paradiso et al., 2013), an effect that has been shown to be even more pronounced in individuals with MDD (Calder, Lawrence, \& Young, 2001; Davis \& Whalen, 2001; Lawrence et al., 2004; Paradiso et al, 2013).

Using a more lenient statistical threshold, additional clusters of hyperactivity were found in the left amygdala, as well as the left nucleus accumbens and right Brodmann areas 46 and 47 within the inferior frontal gyrus. Given the exploratory nature of this analysis, caution is warranted when interpreting these results. The nucleus accumbens is part of the striatum and is considered part of the "reward pathway" as it typically shows activation for rewarding stimuli in healthy controls (Pizzagalli et al., 2009). In individuals with MDD, however, the nucleus accumbens often shows hyperactivation in response to negative stimuli (Monk et al., 2008). Results of the current study also demonstrated hyperactivity in portions of the inferior frontal gyrus that have been considered a pathway between the orbitofrontal cortex, associated with emotion processing, and premotor cortical areas, associated with movement (Cheng et al., 2016; Rolls, 2016; Rolls, 2018; Rolls, 2019; Rolls et al., 2020). These areas have also been hypothesized as ideal targets for therapies like DBS and TMS for treating symptoms of TRD like lack of motivation to perform actions (Pizzagalli et al., 2009; Rolls et al., 2020).

I hypothesized that several regions would show hyperactivity, including prefrontal cortex regions, anterior cingulate cortex, and bilateral amygdala. However, compared to Groenewold et al. (2013), I found fewer areas of hyperactivity. In particular, I did not find the anterior cingulate cortex to be hyperactive. This can likely be explained due to 
using different and more stringent inclusion criteria. In contrast to Gronenewold et al. (2013) in the current study, I excluded papers that used the Monetary Incentive Delay, a task based on decision-making, as those papers did not meet my criteria that the tasks use emotional stimuli. Future studies could run analyses both with and without these tasks (e.g., emotional stimuli and decision-making) to evaluate the impact of this exclusion. Additionally, it is possible that neural hyperactivity could be a form of compensation, allowing individuals with MDD to more effectively engage in daily behavior. More research is needed to determine is needed to determine whether lowering activity levels in these regions results in a behavioral change.

\section{Hypoactivation}

Using a stringent statistical threshold, no regions were found to be consistently hypoactive in MDD relative to controls, but an exploratory analysis revealed hypoactivity in Brodmann area 9 in the right dorsolateral prefrontal cortex in the MDD group. As noted above, results of this exploratory analysis should be interpreted with caution. This finding is generally consistent with symptoms of MDD in that this region is associated with response inhibition; a reduced ability to engage in inhibitory processes could relate to increases in rumination (Aron, Fletcher, Bullmore, Sahakian, \& Robbins, 2003; Picton, 2007). Compared to my hypothesis, I found fewer regions of hypoactivity, likely because of using different and more stringent inclusion criteria.

\section{Stimulus Valence}

The final meta-analysis included only those studies focusing on negative stimuli, whereas the prior analyses included studies that utilized both positive and negative 
stimuli. Results of this narrower analysis indicated that individuals with MDD showed amygdala hyperactivity relative to healthy controls when processing negative stimuli. These findings align with prior reports of exaggerated amygdala reactivity to negative emotional stimuli in individuals with MDD relative to controls (Ramasubbu et al., 2014; Stuhrmann et al., 2013). Other studies suggest that increased activity in the amygdala may also relate to repetitively thinking about or ruminating on negatively salient stimuli (Nejad, Fossati, \& Lemogne, 2013; Whitmer \& Gotlib, 2013).

Although the current study did not investigate neural responses to positive stimuli due to insufficient data, other researchers have demonstrated that individuals with MDD show amygdala hypoactivity relative to controls in response to happy faces (Stuhrmann et al., 2013). Furthermore, this hypoactivity was linked to anhedonia, or an inability to experience pleasure. When more studies are available, future research should involve meta-analyses comparing neural activity in MDD and controls when responding selectively to positive or negative stimuli. Groenewold et al. (2013) found hyperactivity in the medial orbitofrontal cortex in individuals with MDD compared to $\mathrm{HC}$ when processing positive stimuli. Because their inclusion and exclusion criteria were less stringent, they were able to include more studies for the Positive > Neutral analysis.

\section{Clinical Implications}

Current efficacious treatments for MDD include specific psychotherapies and pharmacotherapy; however, $50 \%$ of individuals with MDD do not respond to these treatments. A review of resting-state fMRI studies, or non-task based studies, showed several changes to the brain such as increased connectivity between some regions (e.g., 
middle temporal gyrus to default mode network) and decreased connectivity between others (e.g., prefrontal cortex regions to temporo-limbic regions) when comparing pretreatment and post-treatment images for individuals treated with pharmacotherapies (Dichter et al., 2015). There are no such fMRI studies comparing pre- and post-treatment changes for psychotherapies.

Other, newer treatments include forms of neural stimulation such as DBS and TMS, as well as intranasal ketamine (Pradhan, Parikh, Makani, \& Sahoo, 2015). Preliminary findings from neurostimulation trials have revealed mixed findings in terms of the effectiveness of DBS and TMS (Filkowski \& Sheth, 2019; Wang, 2019). A 2014 metaanalysis of DBS in MDD found that the subgenual cingulate cortex showed the best results when targeted, while a 2010 study found identified the dorsolateral prefrontal cortex as a successfully targeted region (Janicak et al., 2010; Lipsman et al., 2014). Additionally, a 2008 study of TMS found success when targeting the left dorsolateral prefrontal cortex (Drevets, Price, \& Furey, 2008). It bears noting that DBS is a more precise treatment than TMS, given that TMS is non-invasive. Additionally, TMS is wellsuited to stimulating cortical regions on the lateral surface but not deeper cortical or subcortical areas. As such, DSB may be a more effective neurostimulation option for when stimulating the amygdala and ventral portions of the basal ganglia, whereas TMS may be the more appealing option for stimulating the dorsolateral prefrontal cortex.

\section{Limitations}

While powerful, a meta-analytic approach to analyzing common areas of activation across studies does come with limitations. First, although using specific inclusion and 
exclusion criteria eliminated a large number of less relevant studies from the analysis, those that remained still differed from one another in many respects. For example, individual studies differed greatly in the number of participants, mean age of participants, mean length of diagnosis, and mean onset of diagnosis. The stimuli (e.g., words, faces, videos, etc.) and specific tasks (e.g., passive viewing, making emotional ratings, etc.) also differed across studies. It is unclear to what degree these differences may have influenced the results. As more studies on this topic are conducted in the future, new meta-analyses could be performed that allow for greater similarity across studies, e.g., by only including studies that use an emotion rating task.

Second, the meta-analyses evaluating hypoactivity did not return significant results when using a stringent statistical threshold that controlled for multiple comparisons. As such, the exploratory results should be interpreted with caution. Third, in investigating the role of stimulus valence in contributing to neural dysfunction in MDD, I was only able to perform a meta-analysis focusing on negative vs. neutral stimuli given an insufficient number of studies examining positive vs. neutral stimuli. Two meta-analyses comparing neural dysfunction in MDD when processing negative vs. positive stimuli could help elucidate which areas would be most beneficial to target for treatment based on the presentation of symptoms. For instance, studies have demonstrated a link between amygdala hyperactivity in MDD in response to negative stimuli and rumination, and others have shown a relationship between amygdala hypoactivity in response to positive stimuli and anhedonia (Bradley, \& Cuthbert, 1997; Canli et al., 2004; Jenkins et al., 2017; Sacchet et al., 2017). What other regions might show similar patterns? 


\section{Conclusion}

The combined results of these meta-analyses suggest that the amygdala and ventral portions of the basal ganglia are hyperactive in individuals with MDD relative to controls when performing emotion processing tasks. This hyperactivity held when the analysis focused specifically on studies using negative stimuli. Conversely, an exploratory analysis suggests that BA9 (right dorsolateral prefrontal cortex) may be hypoactive in individuals with MDD relative controls. These regions could be targeted when using neurostimulation approaches, such as DBS and TMS, in an effort to improve upon the limited options available in treatment resistant depression. 


\section{References}

Admon, R., Kaiser, R. H., Dillon, D. G., Beltzer, M., Goer, F., Olson, D. P., ... Pizzagalli, D. A. (2016). Dopaminergic enhancement of striatal response to reward in major depression. American Journal of Psychiatry, 174(4), 378-386.

Ahmed, S. P., Bittencourt-Hewitt, A., \& Sebastian, C. L. (2015). Neurocognitive bases of emotion regulation development in adolescence. Developmental Cognitive Neuroscience, 15, 11-25.

Akechi, T., Sugishita, K., Chino, B., Itoh, K., Ikeda, Y., Shimodera, S., ... Kato, T. (2020). Whose depression deteriorates during acute phase antidepressant treatment?. Journal of Affective Disorders, 260, 342-348.

Aron, A. R., Fletcher, P. C., Bullmore, E. T., Sahakian, B. J., \& Robbins, T. W. (2003). Stop-signal inhibition disrupted by damage to right inferior frontal gyrus in humans. Nature Neuroscience, 6(2), 115-116.

Barbas, H. (2007). Flow of information for emotions through temporal and orbitofrontal pathways. Journal of Anatomy, 211(2), 237-249.

Beauregard, M., Paquette, V., \& Le, J. (2006). Dysfunction in the neural circuitry of emotional self-regulation in major depressive disorder. Neuroreport, 17(8), 843-846.

Blakemore, S. J. (2008). The social brain in adolescence. Nature Reviews Neuroscience, 9(4), 267.

Calder, A. J., Lawrence, A. D., \& Young, A. W. (2001). Neuropsychology of fear and loathing. Nature Reviews Neuroscience, 2(5), 352-363.

Canli, T., Zhao, Z., Desmond, J. E., Kang, E., Gross, J., \& Gabrieli, J. D. (2001). An fMRI study of personality influences on brain reactivity to emotional stimuli. Behavioral Neuroscience, 115(1), 33.

Cheng, W., Rolls, E. T., Qiu, J., Liu, W., Tang. Y., Huang, C. C., ... Pu, J. (2016). Medial reward and lateral non-reward orbitofrontal cortex circuits change in opposite directions in depression. Brain, 139(12), 3296-309.

Davidson, R. J., Putnam, K. M., \& Larson, C. L. (2000). Dysfunction in the neural circuitry of emotion regulation - a possible prelude to violence. Science, 289(5479), 591-594.

Davis, M., \& Whalen, P. J. (2001). The amygdala: Vigilance and emotion. Molecular Psychiatry, 6(1), 13-34. 
Dichter, G. S., Gibbs, D., \& Smoski, M. J. (2015). A systematic review of relations between resting-state functional-MRI and treatment response in major depressive disorder. Journal of Affective Disorders, 172, 8-17.

Drevets, W. C., Price, J. L., \& Furey, M. L. (2008). Brain structural and functional abnormalities in mood disorders: Implications for neurocircuitry models of depression. Brain Structure and Function, 213(1-2), 93-118.

Drobisz, D., \& Damborská, A. (2019). Deep brain stimulation targets for treating depression. Behavioural Brain Research, 359, 266-273.

Eickhoff, S.B., Laird, A.R., Grefkes, C., Wang, L.E., Zilles, K., \& Fox, P.T. (2009). Coordinate-based activation likelihood estimation meta-analysis of neuroimaging data: A random-effects approach based on empirical estimates of spatial uncertainty. Hum Brain Mapp, 30, 2907-2926.

Etkin, A., Egner, T., \& Kalisch, R. (2011). Emotional processing in anterior cingulate and medial prefrontal cortex. Trends in Cognitive Sciences, 15(2), 85-93.

Filkowski, M. M., \& Sheth, S. A. (2019). Deep Brain Stimulation for Depression: An Emerging Indication. Neurosurgery Clinics, 30(2), 243-256.

Fonseka, B. A., Jaworska, N., Courtright, A., MacMaster, F. P., \& MacQueen, G. M. (2016). Cortical thickness and emotion processing in young adults with mild to moderate depression: A preliminary study. BMC Psychiatry, 16(1), 38.

Gasquoine, P. G. (2013). Localization of function in anterior cingulate cortex: From psychosurgery to functional neuroimaging. Neuroscience \& Biobehavioral Reviews, 37(3), 340-348.

Ghandili, M., \& Munakomi, S. (2019). Neuroanatomy, Putamen. StatPearls [Internet]. StatPearls Publishing.

Gotlib, I. H., \& Joormann, J. (2010). Cognition and depression: Current status and future directions. Annual Review of Clinical Psychology, 6, 285-312.

Gravetter, F. J., Wallnau, L. B., Forzano, L. A. B., \& Witnauer, J. E. (2020). Essentials of Statistics for the Behavioral Sciences. Cengage Learning.

Groenewold, N. A., Opmeer, E. M., de Jonge, P., Aleman, A., \& Costafreda, S. G. (2013). Emotional valence modulates brain functional abnormalities in depression: Evidence from a meta-analysis of fMRI studies. Neuroscience \& Biobehavioral Reviews, 37(2), 152-163. 
Gross, J. J. (2002). Emotion regulation: Affective, cognitive, and social consequences. Psychophysiology, 39(3), 281-291.

Hamilton, J. P., Siemer, M., \& Gotlib, I. H. (2008). Amygdala volume in major depressive disorder: A meta-analysis of magnetic resonance imaging studies. Molecular Psychiatry, 13(11), 993.

Itagaki, K., Takebayashi, M., Abe, H., Shibasaki, C., Kajitani, N., Okada-Tsuchioka, M., ... Yamawaki, S. (2019). Reduced serum and cerebrospinal fluid levels of autotaxin in major depressive disorder. International Journal of Neuropsychopharmacology, 22(4), 261-269.

Janicak, P. G., Nahas, Z., Lisanby, S. H., Solvason, H. B., Sampson, S. M., McDonald, W. M., ... O'Reardon, J. P. (2010). Durability of clinical benefit with transcranial magnetic stimulation (TMS) in the treatment of pharmacoresistant major depression: Assessment of relapse during a 6-month, multisite, open-label study. Brain Stimulation, 3(4), 187-199.

Jaworska, N., Yang, X. R., Knott, V., \& MacQueen, G. (2015). A review of fMRI studies during visual emotive processing in major depressive disorder. The World Journal of Biological Psychiatry, 16(7), 448-471.

Joormann, J., \& Quinn, M. E. (2014). Cognitive processes and emotion regulation in depression. Depression and Anxiety, 31(4), 308-315.

Kaiser, R. H., Andrews-Hanna, J. R., Spielberg, J. M., Warren, S. L., Sutton, B. P., Miller, G. A., ... Banich, M. T. (2015). Distracted and down: Neural mechanisms of affective interference in subclinical depression. Social Cognitive and Affective Neuroscience, 10(5), 654-663.

Kaiser, R. H., Whitfield-Gabrieli, S., Dillon, D. G., Goer, F., Beltzer, M., Minkel, J., ... Pizzagalli, D. A. (2016). Dynamic resting-state functional connectivity in major depression. Neuropsychopharmacology, 41(7), 1822.

Kisely, S., Li, A., Warren, N., \& Siskind, D. (2018). A systematic review and metaanalysis of deep brain stimulation for depression. Depression and Anxiety, 35(5), 468480.

Laird, A. R., Fox, M., Price, C. J., Glahn, D. C., Uecker, A. M., Lancaster, J. L., ... Fox, P. T. (2005). ALE meta-analysis: Controlling the false discovery rate and performing statistical contrasts. Hum Brain Mapp 25, 155-164.

Laird, A. R., Lancaster, J. L., Fox, M., \& Uecker, A. M. (2003). Users’ Manual for BrainMap GingerALE 2.0. BrainMap. http://brainmap.org 
Lang, P. J., Bradley, M. M., \& Cuthbert, B. N. (1997). International affective picture system (IAPS): Technical manual and affective ratings. NIMH Center for the Study of Emotion and Attention, 1, 39-58.

Lawrence, N. S., Williams, A. M., Surguladze, S., Giampietro, V., Brammer, M. J., Andrew, C., ... Phillips, M. L. (2004). Subcortical and ventral prefrontal cortical neural responses to facial expressions distinguish patients with bipolar disorder and major depression. Biological psychiatry, 55(6), 578-587.

LeDoux, J. E. (2000). Emotion circuits in the brain. Annual Review of Neuroscience, 23(1), 155-184.

Levens, S. M., \& Gotlib, I. H. (2015). Updating emotional content in recovered depressed individuals: Evaluating deficits in emotion processing following a depressive episode. Journal of Behavior Therapy and Experimental Psychiatry, 48, 156-163.

Levy, B. J., \& Wagner, A. D. (2011). Cognitive control and right ventrolateral prefrontal cortex: Reflexive reorienting, motor inhibition, and action updating. Annals of the New York Academy of Sciences, 1224(1), 40.

Lipsman, N., Sankar, T., Downar, J., Kennedy, S. H., Lozano, A. M., \& Giacobbe, P. (2014). Neuromodulation for treatment-refractory major depressive disorder. Cmaj, 186(1), 33-39.

Liu, S., Sheng, J., Li, B., \& Zhang, X. (2017). Recent advances in non-invasive brain stimulation for major depressive disorder. Frontiers in Human Neuroscience, 11, 526.

Matt, G. E., Vázquez, C., \& Campbell, W. K. (1992). Mood-congruent recall of affectively toned stimuli: A meta-analytic review. Clinical Psychology Review, 12(2), 227-255.

Monk, C. S., Klein, R. G., Telzer, E. H., Schroth, E. A., Mannuzza, S., Moulton III, J. L., ... Blair, R. J. (2008). Amygdala and nucleus accumbens activation to emotional facial expressions in children and adolescents at risk for major depression. American Journal of Psychiatry, 165(1), 90-98.

Müller, V. I., Cieslik, E. C., Serbanescu, I., Laird, A. R., Fox, P. T., \& Eickhoff, S. B. (2017). Altered brain activity in unipolar depression revisited: Meta-analyses of neuroimaging studies. JAMA Psychiatry, 74(1), 47-55. 
Murray, C. J., Barber, R. M., Foreman, K. J., Ozgoren, A. A., Abd-Allah, F., Abera, S. F., ... Abu-Rmeileh, N. M. (2015). Global, regional, and national disability-adjusted life years (DALYs) for 306 diseases and injuries and healthy life expectancy (HALE) for 188 countries, 1990-2013: Quantifying the epidemiological transition. The Lancet, 386(10009), 2145-2191.

Neacsiu, A. D., Fang, C. M., Rodriguez, M., \& Rosenthal, M. Z. (2018). Suicidal behavior and problems with emotion regulation. Suicide and Life-Threatening Behavior, 48(1), 52-74.

Nejad, A. B., Fossati, P., \& Lemogne, C. (2013). Self-referential processing, rumination, and cortical midline structures in major depression. Frontiers in Human Neuroscience, 7, 666.

Nemeroff, C. B., \& Owens, M. J. (2002). Treatment of mood disorders. Nature Neuroscience, 5, 1068.

Ochsner, K. N., Bunge, S. A., Gross, J. J., \& Gabrieli, J. D. (2002). Rethinking feelings: An FMRI study of the cognitive regulation of emotion. Journal of Cognitive Neuroscience, 14(8), 1215-1229.

Özyurt, G., Öztürk, Y., Onat, M., Mutlu, C., \& Akay, A. (2018). Attachment, emotion regulation and anger expression in adolescent depression: Did comorbid anxiety disorder not have a role?. Current Psychology, 1-10.

Paradiso, S., Ostedgaard, K., Vaidya, J., Ponto, L. B., \& Robinson, R. (2013). Emotional blunting following left basal ganglia stroke: The role of depression and fronto-limbic functional alterations. Psychiatry Research: Neuroimaging, 211(2), 148-159.

Park, C., Rosenblat, J. D., Lee, Y., Pan, Z., Cao, B., Iacobucci, M., \& McIntyre, R. S. (2019). The neural systems of emotion regulation and abnormalities in major depressive disorder. Behavioural Brain Research.

Phillips, M. L., Drevets, W. C., Rauch, S. L., \& Lane, R. (2003). Neurobiology of emotion perception I: The neural basis of normal emotion perception. Biological Psychiatry, 54(5), 504-514.

Picton, T. W., Stuss, D. T., Alexander, M. P., Shallice, T., Binns, M. A., \& Gillingham, S. (2007). Effects of focal frontal lesions on response inhibition. Cerebral Cortex, 17(4), 826-838. 
Pizzagalli, D. A., Holmes, A. J., Dillon, D. G., Goetz, E. L., Birk, J. L., Bogdan, R., ... Fava, M. (2009). Reduced caudate and nucleus accumbens response to rewards in unmedicated individuals with major depressive disorder. American Journal of Psychiatry, 166(6), 702-710.

Pradhan, B., Parikh, T., Makani, R., \& Sahoo, M. (2015). Ketamine, transcranial magnetic stimulation, and depression specific yoga and mindfulness based cognitive therapy in management of treatment resistant depression: Review and some data on efficacy. Depression Research and Treatment, 2015.

Ramasubbu, R., Konduru, N., Cortese, F., Bray, S., Gaxiola, I., \& Goodyear, B. (2014). Reduced intrinsic connectivity of amygdala in adults with major depressive disorder. Frontiers in Psychiatry, 5, 17.

Rive, M. M., van Rooijen, G., Veltman, D. J., Phillips, M. L., Schene, A. H., \& Ruhé, H. G. (2013). Neural correlates of dysfunctional emotion regulation in major depressive disorder. A systematic review of neuroimaging studies. Neuroscience \& Biobehavioral Reviews, 37(10), 2529-2553.

Rolls, E.T. (2016). A non-reward attractor theory of depression. Neuroscience and Biobehavioral Reviews, 68, 47-58.

Rolls, E.T. (2018). The Brain, Emotion, and Depression, Oxford: Oxford University Press.

Rolls, E.T. (2019). The Orbitofrontal Cortex, Oxford: Oxford University Press.

Rolls, E. T., Cheng, W., Du, J., Wei, D., Qiu, J., Dai, D., ... Feng, J. (2020). Functional connectivity of the right inferior frontal gyrus and orbitofrontal cortex in depression. Social Cognitive and Affective Neuroscience, 15(1), 75-86.

Rosenblau, G., Sterzer, P., Stoy, M., Park, S., Friedel, E., Heinz, A., ... Ströhle, A. (2012). Functional neuroanatomy of emotion processing in major depressive disorder is altered after successful antidepressant therapy. Journal of Psychopharmacology, 26(11), 1424-1433.

Sacchet, M. D., Levy, B. J., Hamilton, J. P., Maksimovskiy, A., Hertel, P. T., Joormann, J., ... Gotlib, I. H. (2017). Cognitive and neural consequences of memory suppression in major depressive disorder. Cognitive, Affective, \& Behavioral Neuroscience, 17(1), 77-93. 
Silvers, J. A., Buhle, J. T., Ochsner, K. N., \& Silvers, J. (2013). The neuroscience of emotion regulation: Basic mechanisms and their role in development, aging and psychopathology. The Handbook of Cognitive Neuroscience, 1, 52-78.

Sippel, L. M., Roy, A. M., Southwick, S. M., \& Fichtenholtz, H. M. (2016). An examination of the roles of trauma exposure and posttraumatic stress disorder on emotion regulation strategies of Operation Iraqi Freedom, Operation Enduring Freedom, and Operation New Dawn veterans. Cognitive Behaviour Therapy, 45(5), 339-350.

Stuhrmann, A., Dohm, K., Kugel, H., Zwanzger, P., Redlich, R., Grotegerd, D., ... Zwitserlood, P. (2013). Mood-congruent amygdala responses to subliminally presented facial expressions in major depression: Associations with anhedonia. Journal of Psychiatry \& Neuroscience: JPN, 38(4), 249.

Stuhrmann, A., Suslow, T., \& Dannlowski, U. (2011). Facial emotion processing in major depression: A systematic review of neuroimaging findings. Biology of Mood \& Anxiety Disorders, 1(1), 10.

van Eijndhoven, P., van Wingen, G., Fernández, G., Rijpkema, M., Verkes, R. J., Buitelaar, J., \& Tendolkar, I. (2011). Amygdala responsivity related to memory of emotionally neutral stimuli constitutes a trait factor for depression. Neuroimage, 54(2), 1677-1684.

Wang, Q., Tian, S., Tang, H., Liu, X., Yan, R., Hua, L., ... Yao, Z. (2019). Identification of major depressive disorder and prediction of treatment response using functional connectivity between the prefrontal cortices and subgenual anterior cingulate: A real-world study. Journal of Affective Disorders, 252, 365-372.

Wang, Y. (2019). Transcranial direct current stimulation for the treatment of major depressive disorder: A meta-analysis of randomized controlled trials. Psychiatry Research, 276, 186-190.

Whitmer, A. J., \& Gotlib, I. H. (2013). An attentional scope model of rumination. Psychological Bulletin, 139(5), 1036.

Yoo, S. S., Gujar, N., Hu, P., Jolesz, F. A., \& Walker, M. P. (2007). The human emotional brain without sleep - a prefrontal amygdala disconnect. Current Biology, 17(20), R877-R878.

Young, K. D., Siegle, G. J., Zotev, V., Phillips, R., Misaki, M., Yuan, H., ... Bodurka, J. (2017). Randomized clinical trial of real-time fMRI amygdala neurofeedback for major depressive disorder: Effects on symptoms and autobiographical memory recall. American Journal of Psychiatry, 174(8), 748-755. 
Young, K. D., Zotev, V., Phillips, R., Misaki, M., Drevets, W. C., \& Bodurka, J. (2018). Amygdala real-time functional magnetic resonance imaging neurofeedback for major depressive disorder: A review. Psychiatry and Clinical Neurosciences, 72(7), 466481.

Zheng, K. Z., Wang, H. N., Liu, J., Xi, Y. B., Li, L., Zhang, X., ... Li, B. J. (2018). Incapacity to control emotion in major depression may arise from disrupted white matter integrity and OFC-amygdala inhibition. CNS Neuroscience \& Therapeutics, 24(11), 1053-1062.

Zotev, V., Krueger, F., Phillips, R., Alvarez, R. P., Simmons, W. K., Bellgowan, P., ... Bodurka, J. (2011). Self-regulation of amygdala activation using real-time fMRI neurofeedback. PloS One, 6(9). 